\title{
The genetic predisposition to and histogenesis of neurofibromas and neurofibrosarcoma in neurofibromatosis
} Type 1

\author{
VINCENT M. RICCARDI, M.D. \\ The Neurofibromatosis Institute, La Crescenta, California
}

\begin{abstract}
The author addresses the issue of neurofibroma classification and implications for treatment. He emphasizes the importance of understanding that not all neurofibromas are the same and that the key differences between the types of neurofibromas involve which portions of the nerve sheath contribute to the distinctive behavior of the different types of lesions.

Endoneurial neurofibromas derive from cellular elements ordinarily restricted to the endoneurium. Perineurial neurofibromas arise within individual fascicles of a nerve and are largely confined thereby, precluding a breach of the epineurium. Epineurial neurofibromas are contained only by the epineurium, and ultimately that portion of the nerve sheath is breached by these lesions.

Whether the perineurium is present or breached becomes the key element for exploiting this approach to neurofibroma origins, behaviors, and treatment, surgical and medical. With respect to surgical treatment, perineurial neurofibromas will have clean planes of dissection about the involved nerve. In contrast, endoneurial and epineurial neurofibromas infiltrate adjacent tissues, leading to surgical challenges. With respect to pharmaceutical approaches, the integrity of the perineurium is likely to prove critical: a specific function of the perineurium is to serve as a barrier to various materials, microbiological or chemical. Thus, drugs that might be effective when the perineurium is absent or rent may be less effective (or not effective at all) if the perineurium is intact, as is expected in cases of perineurial neurofibromas.
\end{abstract}

KEY WORDS - neurofibroma - neurofibromatosis - neurofibrosarcoma • perineurium • Schwann cell

\section{I}

HAVE PREVIOUSLY PROPOSED that the neurofibromas, specifically as we have come to know them in the context of the human disorder NF1, are not necessarily "tumors" in the usual sense. ${ }^{14}$ Rather, I think that they are best understood as dysplasias, aberrant patterns of growth originating in the embryo or at various times after birth. On the basis of this notion, I have elaborated on two approaches to understanding the origin, progression and treatment-surgical and nonsurgical—of neurofibromas. The first of these involves the suggestion that at least some neurofibromas are a manifestation of aberrant wound healing, with a critical role for the mast cell..$^{12,13,15}$ The second approach involves the notion that not all neurofibromas are the same, that they are different in terms of histological characteristics, timing of onset, natural histo-

\footnotetext{
Abbreviations used in this paper: GAP = GTPase-activating protein; GTP = guanosine triphosphate; $\mathrm{NF1}=$ neurofibromatosis Type 1.
}

ry, and other aspects, ${ }^{14}$ ultimately with particular focus on the perineurium.

In this paper I will focus on the details of the second approach, with some emphasis on the neurosurgical relevance. At this point, many experts on NF1 would emphasize that the NF1 gene is a "tumor suppressor gene" and proceed to explain neurofibroma "tumor" formation in terms of the NF1 gene product, neurofibromin, and its role in the p21-ras growth promotion pathways. Focusing on this tumor suppressor function results in the tendency to interpret genetic information in terms of what is currently the most obvious biochemical element of pathogenesis. However, given the very large size of $N F 1$ and its quite incredible conservation during evolution, there must be more to the gene than simply tumor suppression. This conclusion is supported both by the protean nontumor manifestations of the disorder NF1 and the disorder's almost total absence from feral animal populations (although neurofibromas are common among vertebrates). 
Although I will focus on how the genetic abnormalities and the formation of NF1 neurofibromas are interrelated-with a less intense discussion of neurofibrosarcomas-I also wish to emphasize that the NF1 phenotype entails much more than tumors of the central and peripheral nervous systems and, conversely, that NF1 neurofibromas cannot be explained solely in terms of NF1 mutations and abnormal function of neurofibromin. For example, as I pointed out over 20 years ago, mast cells play a key role in the origin and progression of neurofibromas. ${ }^{12,13,15}$

Ultimately, NF1 is a genetic disorder that is particularly efficient at producing neurofibromas. It is one of the most common of all human genetic disorders, with a prevalence of about one in 3000 and a mutation rate of about one in 10,000. Given that there are over a million people with NF1 at any one time, it is not surprising that differences among the innumerable NF1 neurofibromas have become obvious.

Neurofibromatosis Type 1 is an autosomal dominant disorder; approximately one third of cases represent new germinal mutations and a much smaller proportion represent mosaicism due to postzygotic mutations. The occurrence rate among offspring of patients with NF1 is no less than $50 \%$, meaning that penetrance is complete, with no "skipped generations." The expressivity is markedly variable, meaning that from one person to another, even within a family, the manifestations are quite different. Conversely, some mutations seem to have more or less distinctive phenotypes. For example, patients whose mutation is accounted for by deletion of the entire gene (usually the maternal allele) have more problems with learning disabilities, perhaps a distinctive facies and habitus and earlier onset of neurofibromas, involving both the skin and paraspinal regions. ${ }^{10}$ Another group of NF1 patients - those having a deletion of the nucleotide base triplet AAT in exon 17 of $N F 1$ - actually have no neurofibromas involving the skin and very few elsewhere. ${ }^{23}$

The NF1 gene is very large and is located proximally on the long arm of human chromosome 17 at position $17 \mathrm{q} 11.2 .{ }^{26} \mathrm{It}$ is highly conserved, being present, for example, in the fruit fly (Drosophila). The NFI gene is also very complex, with an open reading frame of 8454 nucleotides in about 335 kilobases of genomic DNA and 61 exons. Splice variants are known, and posttranslational modification is a factor as well. This last fact means that expression of $N F 1$ is complex. The gene product, neurofibromin, is a relatively large peptide, with a molecular weight of greater than $220 \mathrm{kD}$. One region of neurofibromin functions as a GAP. The neurofibromin GAP function is one of many within the cell that are involved in the coordination of cellular and tissue physiology. The GAPrelated domain of $N F 1$ selectively stimulates the intrinsic GTPase of p21-ras, which in turn curbs cell proliferation under the control of p21-ras. Loss-of-function mutations in NF1 result in reduced control of cell proliferation. In short, by this description, NF1 is a tumor suppressor gene, although obviously this explanation does not account for all of the functions of either $N F 1$ or neurofibromin or for all aspects of disorders resulting from $N F 1$ mutation. For example, the deletion mutation noted above that leads to NF1 without skin neurofibromas is in exon 17, which is not part of the NF1 GAP-related domain.
Exactly how loss of function of neurofibromin accounts for all of the features of NF1 and neurofibromas in particular is unknown. More specifically, it is not known how one mutation can manifest as multiple types of neurofibromas, each of which has a distinctive natural history and consequence. When loss of the second-normal-allele is sought in cells of NF1-associated tumors, only some neurofibromas and virtually all NF1-associated malignancies (neurofibrosarcomas) show such a deficiency (loss of heterozygosity).

\section{Four Perspectives for Appreciating Subtypes of NF1 Neurofibromas}

\section{The Mature Peripheral Nerve Sheath, Dorsal Root Ganglia, and Rootlets}

The peripheral nerve sheath has three main elements, the epineurium, the perineurium, and the endoneurium. Understanding these three tissue components properly requires appreciation of both the final nerve structure and the developmental processes leading to that structure.

In the mature nerve, the epineurium is the outermost membrane, literally ensheathing the entire nerve distal to the dorsal root ganglion. It is composed of fibroblasts, adipocytes, endothelial cells, mast cells, and large amounts of collagen. ${ }^{19,24}$ The epineurium also projects inward to surround the individual nerve fascicles that are defined by the perineurium. The epineurium separates the nerve from surrounding tissues and provides a mechanical cushion, particularly at branch points and passages through osseous foramina.

In the mature nerve, the perineurium surrounds and defines the individual nerve fascicles within the nerve delineated by the epineurium. It consists of flattened perineurial cells and small amounts of dense collagen. The perineurial cells are characterized by their flattened appearance, being stacked in layers four to 12 cells deep, the presence of a basement membrane and, visualized with electron microscopy, having numerous pinocytic vesicles. The perineurium also protrudes centrally to subdivide each fascicle. Smaller and more distal nerve fascicles have smaller numbers of associated perineurial cells. There are two primary functions of the perineurium: providing the tensile strength of the nerve $\mathrm{e}^{20-22}$ and providing a microbiological and biochemical nerve-blood barrier.

In the mature nerve, the endoneurium is confined by the perineurium, and its main components include multiple axons and their respective associated Schwann cells, with or without myelination, as well as neural-crestderived fibroblasts, ${ }^{11}$ endothelial cells, mast cells, and small amounts of collagen. In the termini of sensory nerves (at least those in the skin), there is no endoneurium, as the axon extends beyond the limits of the epineurium and the perineurium.

The mature dorsal root ganglion contains the cell bodies of the sensory nerves. Each ganglion cell has an associated so-called satellite cell, considered to be a form of Schwann cell, as well as axonal projections extending centripetally into the spinal cord and centrifugally to form the sensory components of peripheral nerves. Each axon has its associated Schwann cells, with or without myelination. 
Centrally, the dorsal root ganglion axons and their Schwann cells are bundled into small rootlets (the dorsal nerve rootlets), invested proximally by a nonspecific layer of fibroblasts and collagen until the dura is pierced, whereupon the rootlets are invested by the pia mater and arachnoid membrane. Upon entering the spinal cord, the axons project cephalad to their final intraspinal junctions.

\section{Embryology of the Peripheral Nerve Sheath, Dorsal Root Ganglia, and Rootlets}

The neural crest first appears as a sheet of ectodermderived cells spread over the dorsum and upper sides of the developing spinal cord. This sheet coalesces ultimately to form discrete ganglia, including both the paraspinal sympathetic ganglia and the paraspinal dorsal root ganglia corresponding to each somite. (The neural crest contributes to many other tissues and cell types, including melanocytes, chromaffin cells, skeletal components of the head and face, and others, but they will not be pursued here.)

Each dorsal root ganglion cell becomes associated with its own Schwann-cell-like satellite cell, also derived from the neural crest. As each ganglion cell differentiates and grows, it sends axonal projections centrally toward the spinal cord, ultimately to form synapses within the cord, and peripherally to innervate local and distal tissues. Each axon is ensheathed in one or more Schwann cells along its entire length. As the axon grows, the Schwann cells proliferate. Some axons become myelinated, while others do not.

At first the axons and their associated Schwann cells emanating from an individual ganglion form a simple, relatively homogeneous bundle surrounded only by a nonspecific connective tissue membrane (fibroblasts and collagen). As axon growth progresses, the Schwann cells multiply and synthesize, posttranslationally modify, and secrete the protein known as desert hedgehog, leading in turn to the induction and maturation of perineurial cells, which eventually subdivide the nerve bundle into discrete fascicles of varying size. Each fascicle ultimately is circumscribed by a well-defined perineurium as described previously. As the immature nerve progresses in its growth, however, the sheets of cells that ultimately form the mature perineurium form an incomplete membrane. The perineurium, for approximately a 2-week period of its development, is, so to speak, fenestrated. The integrity required in order to provide the properties of high tensile strength and diffusion barrier will be attained a bit later, a fact potentially of particular relevance to the origin of one form of neurofibroma.

\section{The Pathology and Biology of Neurofibromas}

A neurofibroma is a mass that contains multiple cell types-Schwann cells, perineurial cells, nonspecific fibroblasts, endoneurial fibroblasts, endothelial cells, mast cells, and sometimes adipocytes, smooth muscle cells, melanocytes, and hair cells-and as well large amounts of intercellular material, including collagen and amorphous ground substance, for example, mucopolysaccharides and glycosaminoglycans. The details of the histopathology and biochemistry of neurofibromas have been most carefully characterized by Jaakkola and colleagues. ${ }^{6}$ In any event, the histopathological characteristics of neurofibromas have been considered to be the same, regardless of what type of neurofibroma is being studied.

Most people would immediately refer to a neurofibroma as a tumor. I would not. I prefer to consider a neurofibroma in more neutral terms. Yes, it is a mass that enlarges over time: a neurofibroma has that in common with tumors, more strictly defined. Consider, on the other hand, a keloid, which is not ordinarily referred to as a tumor. Rather, it is a circumscribed collection of cells and intercellular material that accumulate in response to trauma. I consider this description to explain at least some types of neurofibromas. A neurofibroma is a mass that represents coordinated hyperplasia, not simply the clonal proliferation of one cell type.

A schwannoma is such a clonal proliferation. A schwannoma is a monotonous accumulation of Schwann cells, with very little intercellular material. It arises within a single fascicle and imposes on the rest of the nerve more or less as an external mass. In contrast, a neurofibroma arises within the substance of a nerve such that it is an intrinsic part of the nerve. Surgical removal of a neurofibroma requires sacrifice of portions of the nerve. Surgical removal of a schwannoma can leave the nerve otherwise intact. A schwannoma is a tumor in the ordinary sense. A neurofibroma is not. Again, I prefer to think of a neurofibroma as disordered hyperplasia, perhaps even an aberrant response to trauma. Schwannomas do not occur in NF1, but they do occur in NF2 and multiple schwannomatosis.

The Schwann cell has been an important focus of neurofibroma pathogenesis for several reasons. First, these cells are the most readily identifiable cells of neural crest origin contributing to neurofibromas. Second, Schwann cells grow readily in laboratory cell culture systems, in contrast, for example, to perineurial cells. In short, they are easy to study. Third, in another so-called neurofibromatosis, NF2, one of its prototypic tumors, the schwannoma, is composed almost entirely of Schwann cells. The fibroblast also grows readily in cell cultures of neurofibromas, although it has summarily been dismissed as being merely a "contaminant," a bystander or a passive respondent. In any event, the neurofibroma-associated fibroblast is presumed, incorrectly, not to be of neural crest origin. ${ }^{8}$ The perineurial cell has been given relatively short shrift as part of neurofibroma pathogenesis, with most data dependent on and derived from ultrastructural depictions of very small portions of any given lesion. (Very often note is merely made of the presence of perineurial cells, without specification of whether they are part of an intact perineurium.) In a way, the perineurium has been hiding in plain sight, obscured in a sense by the focus on Schwann cells.

Neurofibromas are relatively well known among all classes of jawed vertebrates, ${ }^{14,17}$ including chondrichthyes (sharks and rays) and osteichthyes, for example, the goldfish and the bicolor damselfish (Pomacentrus partitus). Various species of birds manifest neurofibromas. Among mammals, a wide variety of domestic animals, including dogs and cattle, develop isolated neurofibromas. Nonetheless, no disorder resembling NF1 in any way is known among feral mammals. In my opinion, this is a critically important indication that the NFI gene involves more than 
merely neurofibroma formation. Laboratory-induced neurofibromas in mice and rats are well known, particularly consequent to in utero exposure to the mutagen ethylnitrosourea. ${ }^{1,25}$ Murine counterparts to NF1 have been developed several times in mice by genetic engineering to pro selected cell types. ${ }^{7,28}$ The so-called neurofibromas in these latter laboratory models are different in multiple ways from the human neurofibroma. For example, in the selective knockout model devised by Zhu and colleagues, ${ }^{28}$ the neurofibromas appear to originate primarily in the dorsal root ganglion and only rarely in the skin, as is seen in human NF1. On the other hand, consistent with what is expected from human NF1, Zhang and associates ${ }^{27}$ have documented that the knockout mouse neurofibroma with loss of both copies of NF1 (loss of heterozygosity) recruits mast cells heterozygous for the knockout, presumably a stage on the path to developing a neurofibrosarcoma.

In humans, neurofibromas occur in isolation or as part of the disorder NF1 (originally known as Von Recklinghausen disease; Online Mendelian Inheritance in Man 162200). There appear to be four clinical types of human neurofibromas that may be classified in three pathogenetic categories, as described in detail in the next section.

\section{The Clinical Perspective for Appreciating Subtypes of NF1 Neurofibromas}

If we go beyond the histopathology of neurofibromas, the story becomes more complicated. Sameness gives way to diversity. There are at least four different clinical types of neurofibromas: ${ }^{14}$ cutaneous, subcutaneous, nodular plexiform, and diffuse plexiform (Table 1). However, for the purposes of understanding pathogenesis, subcutaneous and nodular plexiform neurofibromas can be considered to be the same in an important sense, facilitating delineation of three basic types of neurofibromas: endoneurial, perineurial, and epineurial (Table 2).

My appreciation of this categorization is based on multiple evaluations of over 1200 patients who had or were at risk for NF1, NF2, or the other types of $\mathrm{NF}^{14}$ both through the Baylor Neurofibromatosis Program (1977-1990) and The Neurofibromatosis Institute (1981-present). Data pertaining to many of these cases are also available through the Neurofibromatosis International Database overseen by Dr. J.M. Friedman. ${ }^{3}$ The schema described here is compatible with other demonstrated or potential neurofibroma subtypes, such as "glandular" neurofibroma or "atypical" neurofibroma.

Use of the term "dermal," as in "dermal neurofibromas," is not useful, as three of the four types of neurofibromas involve the dermis one way or another. Simply referring to "plexiform" neurofibromas ${ }^{5}$ fails to reflect their different biological behaviors, with special regard to the epineurium. Both types of plexiform neurofibroma have a "plexus" (braided or network) element, but they are otherwise very different from each other.

\section{The Nature of the Neurofibroma Nerve Sheath Classification}

\section{The Three Types of NF1 Neurofibromas}

The pathogenetic schema for categorizing the three types of NF1 neurofibromas is illustrated in Figs. 1 through 4. The merging of this mechanistic schema with clinical data leads to the recognition that there are actually three basic types of neurofibromas (endoneurial, perineurial, and epineurial) that readily accommodate the four types suggested on purely clinical grounds. Although the clinical perspective still has some utility (for example, accurately predicting the presence of paraspinal neurofibromas if subcutaneous neurofibromas are present), the pathophysiology and treatment strategies are best served by the truncated three-subtype version.

Endoneurial Neurofibromas. Cutaneous neurofibromas are endoneurial neurofibromas. They occur in the skin; if one moves the skin, the neurofibroma will move with the skin. The endoneurial neurofibroma designation derives from the fact that such masses show no evidence of perineurial cells or components identifiable as epineurium: only the Schwann cells and fibroblasts of the endoneurium appear to contribute directly from the involved nerve. It is as though the sensory nerve terminus is incontinent of endoneurial Schwann cells and fibroblasts. This picture is consistent with the work of Karvonen et al., ${ }^{9}$ who identified clusters of S100-positive Schwann cells around skin hair bulbs in ostensibly normal skin of youngsters with NF1. The endoneurial neurofibroma thus arises beyond the confines of the perineurium and the external limit of the nerve, the epineurium, infiltrating and compromising adjacent tissues.

A typical endoneurial neurofibroma (Fig. 5) initially appears as a flat localized reddish to violaceous discoloration. It gradually expands in diameter and becomes protuberant, forming a modest sessile mass. As the neurofibroma grows, its apex protrudes farther and farther from the surface of the normal skin and eventually develops a stalk, giving the mass a pedunculated or polypoid appearance. Both the sessile and pedunculated neurofibromas have a very distinctive quality, referred to as "buttonholing." When one presses on the neurofibroma with a finger, it seems to "pop" through a defect in the underlying connective tissue, as though it had been pushed through a buttonhole. When pedunculated, these masses range in size from about 5 to $30 \mathrm{~cm}$ or more in diameter. Most often they first become manifest just prior to the onset of puberty, actually probably not reflecting puberty per se, but rather the accumulation of body fat that is necessary for puberty to commence. They may arise almost anywhere on the body, although there are some topographical influences, which are discussed later in this section. I have seen adults with as few as a half dozen endoneurial neurofibromas and individuals of the same age range with hundreds or thousands. When the skin becomes carpeted with these masses, skin hygiene becomes difficult and a distinctive odor is readily apparent. (Von Recklinghausen commented on this fact is his original report of an autopsy performed in such a case.) It is not possible to predict the burden of endoneurial neurofibromas based on a childhood and adolescence with no or few endoneurial neurofibromas. On the other hand, an early onset of endoneurial neurofibromas is a harbinger of a greater burden. At least one form of NF1, due to a distinctive mutation, has been described wherein there is a total absence of endoneurial neurofibromas, although epineurial neurofibromas may be present. ${ }^{22}$

The following points emphasize the uniqueness of 
TABLE 1

Clinical classification of NF1 neurofibromas*

\begin{tabular}{|c|c|c|c|c|}
\hline \multirow[b]{2}{*}{ Characteristic } & \multicolumn{4}{|c|}{ Neurofibroma Type } \\
\hline & Cutaneous & Subcutaneous & Nodular Plex & Diffuse Plex \\
\hline \multicolumn{5}{|l|}{ on inspection/palpation } \\
\hline no. of lesions & med-many & few-med & few-med & few \\
\hline lesion size & small-med & small-med & med-large & med-massive \\
\hline location of lesions & trunk & limbs/girdles & paraspinal & anywhere \\
\hline localized & yes & yes & entire nerve & diffuse \\
\hline skin movement & w/ lesion & over lesion & NA & w/ lesion \\
\hline lesion consistency & soft & firm & firm & soft \\
\hline skin discoloration & red/violet & no & NA & brown \\
\hline buttonholing & yes & no & NA & no \\
\hline special & $\mathrm{F}$ areolae & sentinel & paresis & massive \\
\hline \multicolumn{5}{|l|}{ symptoms } \\
\hline pain, tenderness & no & yes & yes & possible \\
\hline itching & yes & no & no & yes \\
\hline nerve size & smallest & small-med & med-large & med-large \\
\hline gross anatomy & bud/infil & nodular & nodular & bud/infil \\
\hline encroachment & replacement & displacement & displacement & replacement \\
\hline clinically obvious & 2nd decade & 1st decade & 1st-3rd decade & congenital \\
\hline natural history & slow & moderate & rapid & slow \\
\hline prognosis & cosmetic & poor & poor & poor \\
\hline sarcoma risk & doubtful & uncertain & high & high \\
\hline environment & trauma & uncertain & uncertain & uncertain \\
\hline
\end{tabular}

* bud = budding $;$ infil = infiltrative med = medium $;$ NA = not applicable Plex = Plexiform .

endoneurial neurofibromas, differentiating them from perineurial neurofibromas and epineurial neurofibromas. As well, at least some of them offer potential insights into additional factors contributing to the origin and progression of this type of neurofibroma in general and specifically emphasize that pathogenesis cannot be accounted for merely in terms of $N F 1$ being a tumor suppressor gene.

"Miliary" Endoneurial Neurofibromas. These neurofibromas occur in a subgroup of patients with NF1, almost always in the later teenage years or early adulthood. There is a virtual eruption over a period of weeks of innumerable endoneurial neurofibromas over the trunk from the clavicles to a line about six centimeters below the umbilicus. In my experience, the vast majority of patients with miliary endoneurial neurofibromas have been young women. I am uncertain whether the long-term natural history of the disease differs in these cases from cases of "ordinary" endoneurial neurofibromas.

The Effect of Pregnancy. During pregnancy the obviousness of endoneurial neurofibromas is typically increased. I doubt, however, that pregnancy itself causes an increase either in the number or the literal growth of these masses. Having observed in detail a series of women progressing through pregnancy and delivery (and documenting these cases with photographs), it seems to me that the endoneurial neurofibromas that are already present become erythematous and swollen, engorged with fluid or ground substance. These phenomena occur over a period of weeks or days and then resolve over an even shorter period of time. This is an important lesson: an increase in the size of the mass does not equate to cellular proliferation. Thus, when we speak about "enlarging" neurofibromas, we must not conclude that the enlargement is necessarily due to cellular proliferation, clonal or otherwise.
The Effect of Fat. Pregnancy is a time for the accumulation of fat in preparation for lactation. Fat accumulation may be a factor accounting for the changes in neurofibromas during pregnancy. The onset of puberty requires a similar prior accumulation of fat. This latter fact fits with my observation that the earliest onset of endoneurial neurofibromas precedes puberty by months. It is as though the cutaneous accumulation of fat contributes to the origin

TABLE 2

Pathogenetic classification of NF1 neurofibromas

\begin{tabular}{|c|c|c|c|}
\hline \multirow[b]{2}{*}{ Characteristic } & \multicolumn{3}{|c|}{ Neurofibroma Type } \\
\hline & Endoneurial & Perineurial & Epineurial \\
\hline \multicolumn{4}{|l|}{ on inspection/palpation } \\
\hline no. of lesions & med-many & few-med & few \\
\hline lesion size & small-med & small-large & med-massive \\
\hline location of lesions & trunk & $\begin{array}{l}\text { limbs/trunk/ } \\
\text { paraspinal }\end{array}$ & anywhere \\
\hline localized & yes & yes/entire nerve & diffuse \\
\hline skin movement & w/ lesion & over lesion & w/ lesion \\
\hline lesion consistency & soft & firm & soft \\
\hline skin discoloration & $\mathrm{red} /$ violet & no & brown \\
\hline buttonholing & yes & no & no \\
\hline special & $\mathrm{F}$ areolae & sentinel/paresis & massive \\
\hline \multicolumn{4}{|l|}{ symptoms } \\
\hline pain, tenderness & no & yes & possible \\
\hline itching & yes & no & yes \\
\hline nerve size & smallest & small-large & med-large \\
\hline gross anatomy & bud/infil & nodular & bud/infil \\
\hline encroachment & replacement & displacement & replacement \\
\hline clinically obvious & 2nd decade & 1st-3rd decade & congenital \\
\hline natural history & slow & moderate-rapid & slow \\
\hline prognosis & cosmetic & poor & poor \\
\hline sarcoma risk & doubtful & high & high \\
\hline environment & trauma & uncertain & uncertain \\
\hline
\end{tabular}




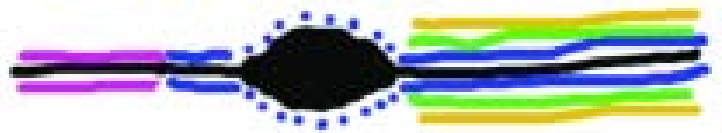

FIG. 1. Sketch depicting a mature dorsal root ganglion cell and its proximal and distal projections and their accompaniments. The black ellipse represents the ganglion cell body and the black lines the axonal projections, proximal to the left side of the image and distal to the right. The blue dots represent the satellite cell components attached to the ganglion cell body. The solid blue line represents Schwann cell components. The green line represents perineurium (which actually would encompass multiple axons, grouping them into fascicles). The yellow lines represent the epineurium (which actually would encompass multiple fascicles). The pink lines represent the combination of pia mater and arachnoid membrane lining the projection once it is internal to the dura mater.

and early progression of endoneurial neurofibromas. Here I also give weight to the observation that endoneurial neurofibromas do not occur on the shins (specifically where the overlying skin has minimal fat) unless fat is accumulated there.

Pruritus. Endoneurial neurofibromas may become intensely itchy, compromising sleep and other activities of daily life. The itchiness appears to be more pronounced during periods of apparent neurofibroma growth. Excessive itchiness associated with enlarging and/or numerous endoneurial neurofibromas was one of the earliest clinical clues to the role of the mast cell in neurofibroma pathogenesis. The mast cell blocker, ketotifen, has been shown to be effective in treating this symptom..$^{14,15}$

The Effect of Trauma. Multiple patients have shared with me the story of intense crush trauma (for instance, a horse bite or closing a body part in a car door) followed by intense pruritus and then the appearance and moderately rapid progression of an endoneurial neurofibroma. Among several patients who have undergone skin transplant surgery, the skin graft sites have shown an excess of endoneurial neurofibromas compared to the surrounding skin.

Coalescence. As endoneurial neurofibromas that are close to each other grow, their adjacent borders may merge, allowing the coalesced mass to seem to be a diffuse plexiform (epineurial) neurofibroma, with its attendant risks.

Anatomical Specificity. The trunk, from the axillary folds to about $6 \mathrm{~cm}$ below the umbilicus, is the site of the greatest numbers of endoneurial neurofibromas. The adult female nipples and areolae virtually always manifest these neurofibromas. There is something about this highly sensitive vascular erectile tissue that predisposes to endoneurial neurofibroma formation. The shins-where there is almost no subcutaneous fat-are consistently spared the presence of endoneurial neurofibromas. Likewise for the glans penis and the vermillion border of the upper lip.

Involution. Occasionally an endoneurial neurofibroma-particularly one on the trunk-will undergo spontaneous involution, leaving behind a hypopigmented atrophic scar. ${ }^{11}$ In at least one case of NF1, denervation has

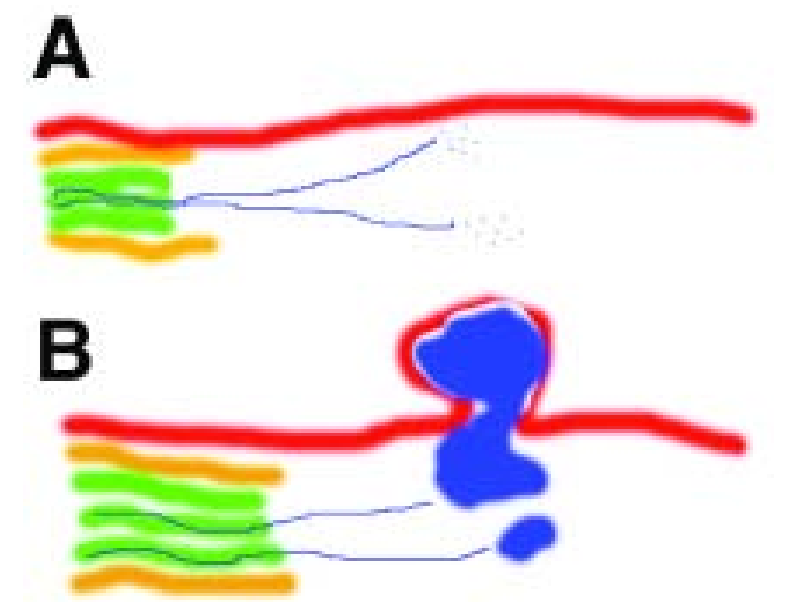

FIG. 2. Sketches depicting two stages in the development of an endoneurial neurofibroma. The orange lines represent the outermost elements of the cutaneous portion of the skin. A: In the earlier stage, the Schwann-cell-ensheathed terminal axons (blue lines) extend beyond the confines of the perineurium and epineurium. The small blue dots represent incontinent Schwann cells, that is, Schwann cells that have detached from the axon and situated themselves in the epidermis or dermis. B: In the later stage, the incontinent Schwann cells have progressed to form a neurofibroma (solid blue masses, the "Zapfen" of von Recklinghausen's original description of endoneurial neurofibromas), one portion of which has protruded through and attenuated the overlying epidermis.

been reported to lead to regression of endoneurial neurofibromas within the denervated skin. ${ }^{16}$

Malignancy. Endoneurial neurofibromas are not thought to evolve into neurofibrosarcomas, in contrast to neurofibromas of either the perineurial or epineurial type.

Surgical Considerations. Removal of endoneurial neurofibromas by surgery, whether with a steel blade or laser, is generally discouraged unless the mass is particularly intrusive functionally or in terms of cosmesis. For example, plantar neurofibromas can become painful or compromise fitting a foot into a shoe; chest wall neurofibromas may become irritated from a brassiere. Unless the excision is particularly wide, some portion will be left behind and some degree of regrowth will be likely, although the regrowth is not likely to be exaggerated - that is, the trauma of surgery is not apparently a stimulus to neurofibroma enlargement. ${ }^{4}$

Perineurial Neurofibromas. Subcutaneous and nodular plexiform neurofibromas are perineurial fibromas. Pathogenetically, perineurial neurofibromas are characterized by confinement of most or all of the mass to individual nerve fascicles by virtue of an intact perineurium. Beyond the perineurium the epineurium is intact as well, providing a clean plane of dissection around the involved nerve. This is utterly distinct from the endoneurial neurofibromas already discussed and the diffuse plexiform or epineurial neurofibromas to be discussed later in this article. Multiple fascicles may be involved at discrete locations along the nerve or throughout the entire length of the nerve, starting from the paraspinal dorsal root region.

Subcutaneous neurofibromas are locally discrete perineurial neurofibromas-simplex perineurial neurofibro- 


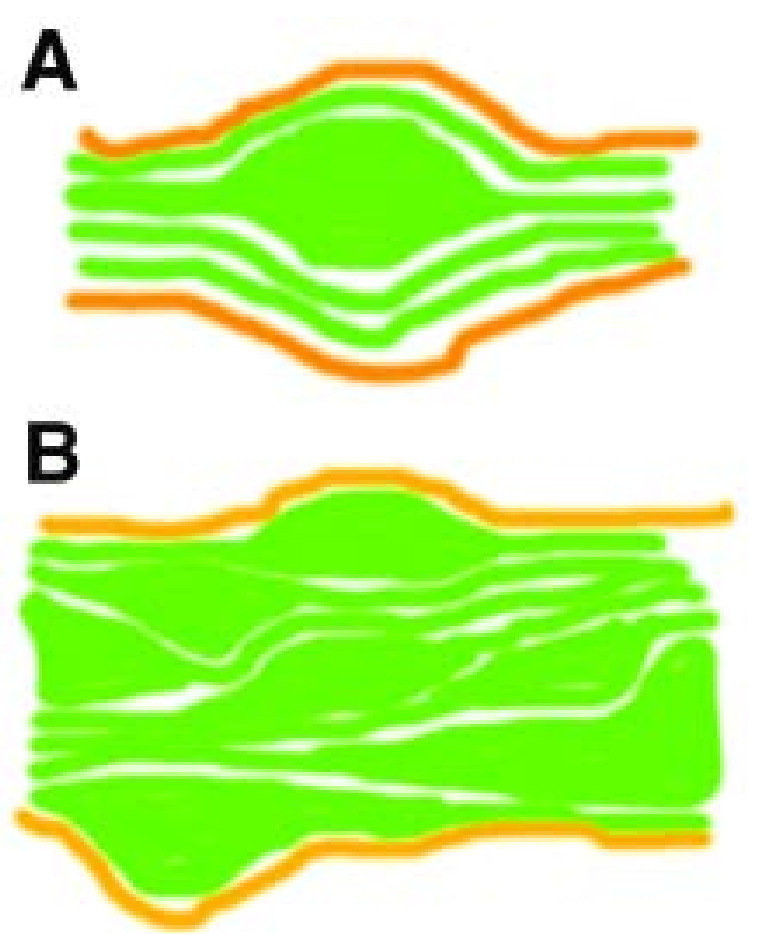

FIG. 3. Sketches depicting two subtypes of perineurial neurofibroma. The yellow lines represent the epineurium. A: A simplex perineurial neurofibroma. The neurofibroma (green ellipse) is contained within the perineurium, defining one distinct fascicle. Other fascicles (without neurofibromas) are represented by the green lines. B: A multiplex perineurial neurofibroma, actually a composite of simplex perineurial neurofibromas (green ellipses) at different locations along the length of the nerve.

mas - that are actually under the skin: when one moves the skin, it moves over the neurofibroma. This is easily appreciated on the volar surface of the forearm where subcutaneous neurofibromas may feel like rosary beads as one passes one's fingers over the skin from the crux of the elbow to the skin creases at the wrist. They do not extend into and replace adjacent tissue; rather, they simply displace it (Fig. 6).

Nodular plexiform neurofibromas occur on dorsal nerve roots along the spinal column and on large and medium-sized nerves-multiplex perineurial neurofibromas. If they are present at birth, they are subtle, too subtle even to be recognized by ordinary clinical means during infancy and early childhood. Once obvious, however, they have a distinctive appearance on gross dissection, causing an involved nerve to look like a string or rope that has been twisted or coiled excessively. It appears to be knotted; hence, the term nodular. (This description fits with von Recklinghausen's appellation of "Rankenneurome" or "twisted neuroma.") A nerve that ordinarily might be the size of a pencil lead will assume the size of the pencil itself, albeit knotted and distorted. Multiplex perineurial neurofibromas are almost always painful and tender. They also erode adjacent osseous structures, particularly vertebrae. Vertebral erosion by nodular plexiform neurofibromas is a common cause of death among patients that have these neurofibromas: the vertebral erosion leads to com-
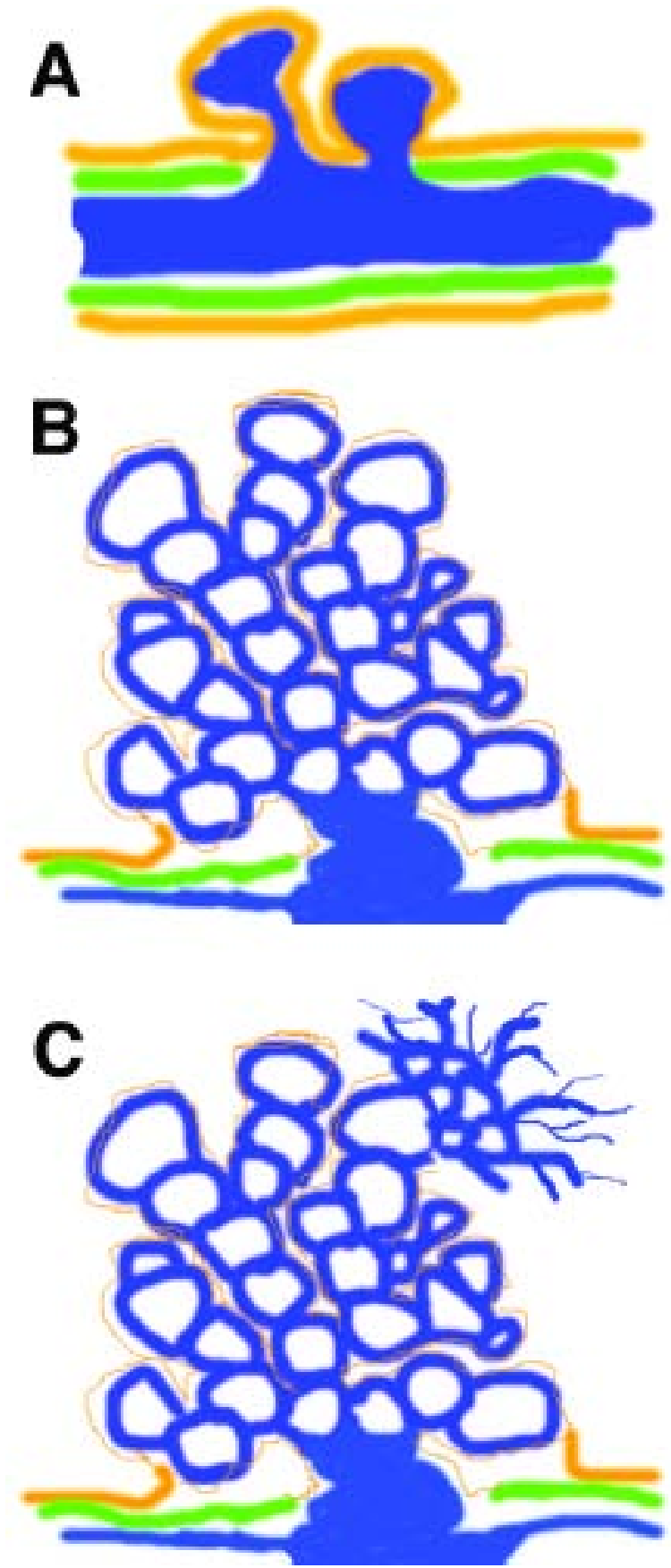

FIG. 4. Sketches depicting three stages of an epineurial neurofibroma. A: Breached perineurium. The thick blue line represents a fascicular neurofibroma (and its associated Schwann cells) that has breached the perineurium and protrudes outward, displacing the epineurium (yellow lines). B: Attenuated epineurium. The circular blue lines represent the budding (growing) neurofibroma as it attenuates the surrounding epineurium (thin yellow lines), displacing adjacent normal tissue. C: Breached epineurium. Components (thin blue lines) of one bud have breached the epineurium and are enmeshed in and infiltrating the adjacent tissue.

pression of the spinal cord and loss of critical enhancement of respiration. Patients with multiplex perineurial neurofibromas usually die either from asphyxiation due to 


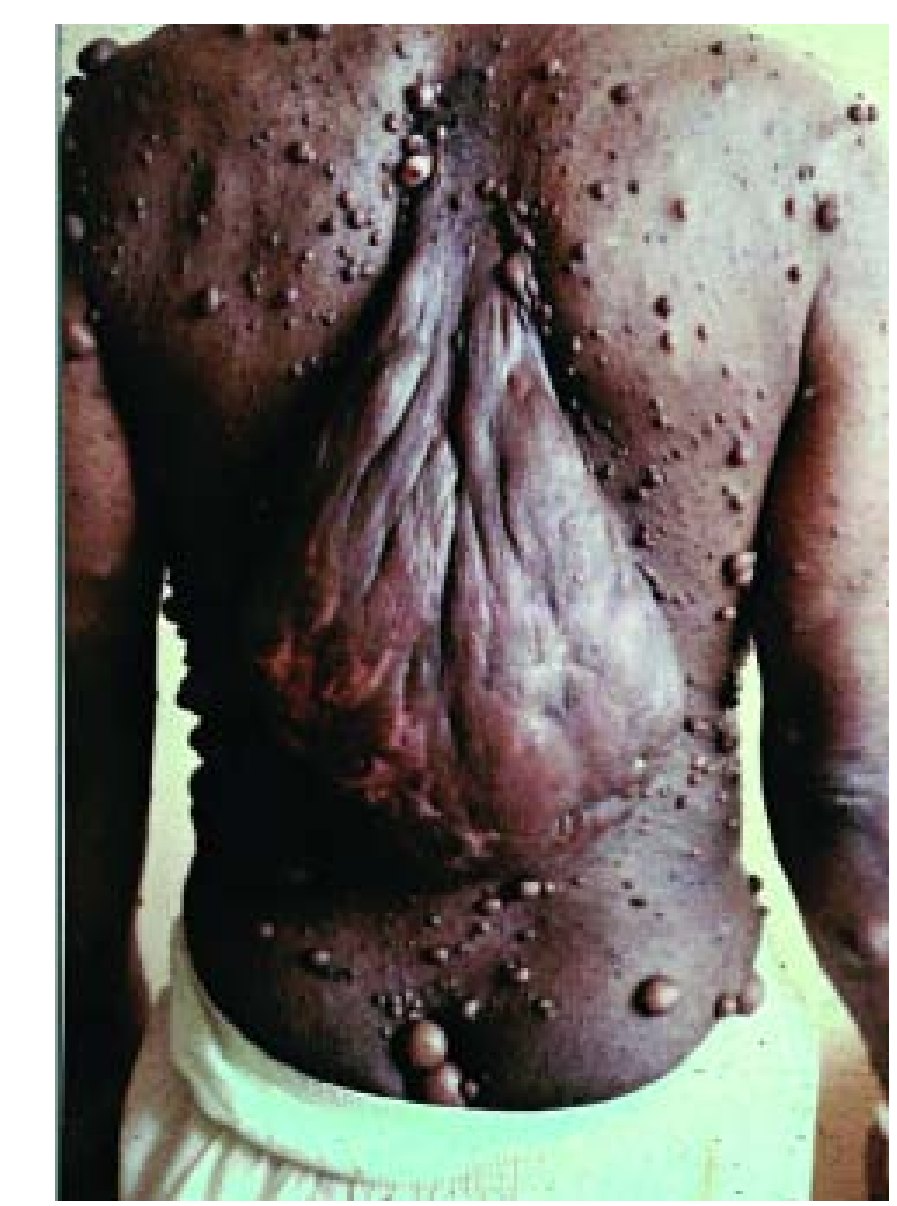

FIG. 5. Photograph of the posterior trunk of a man showing endoneurial neurofibromas in various stages of maturation.

spinal cord compression or from metastases consequent to malignant transformation of the neurofibroma.

The points emphasize the uniqueness of perineurial neurofibromas, differentiating them from endoneurial neurofibromas and epineurial neurofibromas. As well, at least some of them offer potential insights into additional factors contributing to the origin and progression of this type of neurofibroma in general and specifically emphasize that pathogenesis cannot be accounted for merely in terms of NF 1 being a tumor suppressor gene.

The Role of Neuroimaging. Magnetic resonance imaging studies of the spine at all levels should be performed in either of two settings: if a paraspinal neurofibroma is found at any level or if the patient has multiple simplex perineurial neurofibromas. All dorsal roots may be involved.

Multiple Simplex Perineurial Neurofibromas. The presence of multiple simplex perineurial neurofibromas should always prompt an evaluation for paraspinal multiplex perineurial neurofibromas.

Association With Deletion of the NF1 Gene. Perineurial neurofibromas appear to be more frequent among patients with NF1 due to whole gene deletions.

Negative Consequences of Surgery. Surgical treatment

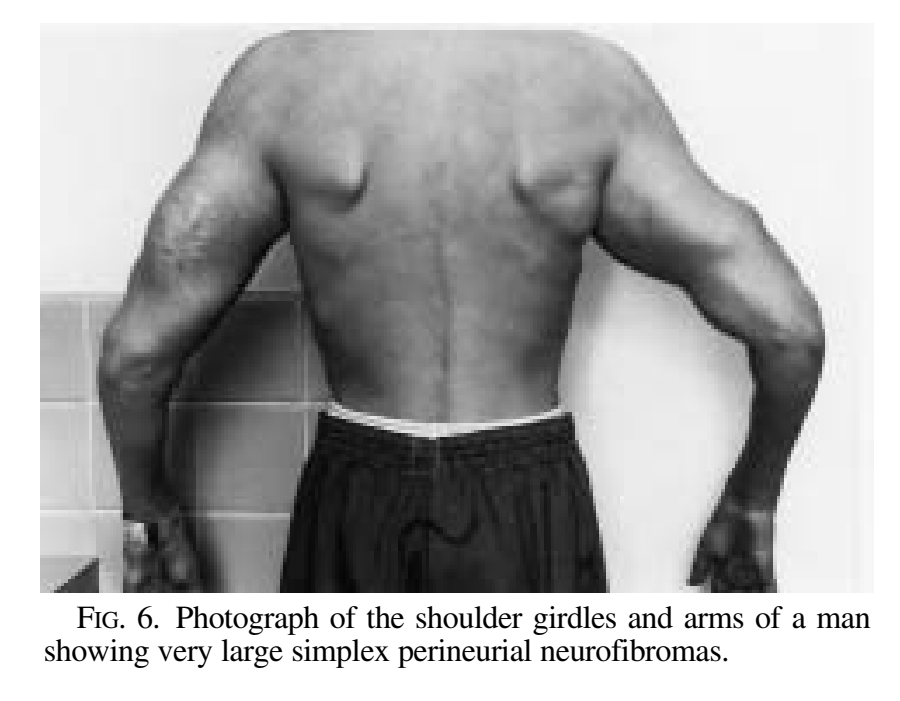

of perineurial neurofibromas seriously hazards significant postoperative neurological compromise.

Neurological Deficits. Even without surgery, the likelihood of severe sensory and motor deficits developing is very high.

Risk of Neurofibrosarcoma. The probability of a neurofibrosarcoma developing in patients with perineurial neurofibroma is greater than $10 \%$.

Epineurial Neurofibromas. Diffuse plexiform neurofibromas are epineurial neurofibromas. These lesions involve-or come to involve-all of the tissues that surround them. They are not simply "nerve tumors."

Epineurial neurofibromas are congenital in origin. Considering their congenital nature and the absence of an intact perineurium in or around these neurofibromas, I suspect that they arise during that period of embryological peripheral nerve development when the perineurium is still fenestrated.

To the extent that this type of neurofibroma is still contained in the nerve of origin, it is bound only by the epineurium, which itself is also eventually breached by attenuation and direct invasion. Upon breaching the epineurium, the epineurial neurofibroma literally diffuses into adjacent normal tissue, ultimately replacing it. The nerve and the neurofibroma are enmeshed in the surrounding tissue, some of which is normal for the site or has already undergone varying degrees of metaplasia, often into adipocytes. This makes the identification of anatomical landmarks critical for surgery extremely difficult.

Thus, the epineurial neurofibroma is a dysplasia-an aberration of growth-both during embryogenesis and subsequently. When there is overlying hyperpigmentation and hypertrichosis, the dysplasia is unequivocally obvious. Otherwise, the dysplasia may not be so blatant. The dysplasia is present nonetheless.

The following points emphasize the uniqueness of epineurial neurofibromas, differentiating them from endoneurial neurofibromas and perineurial neurofibromas. As well, at least some of them offer potential insights into additional factors contributing to the origin and progression of this type of neurofibroma in general and again specifically emphasize that pathogenesis cannot be ac- 


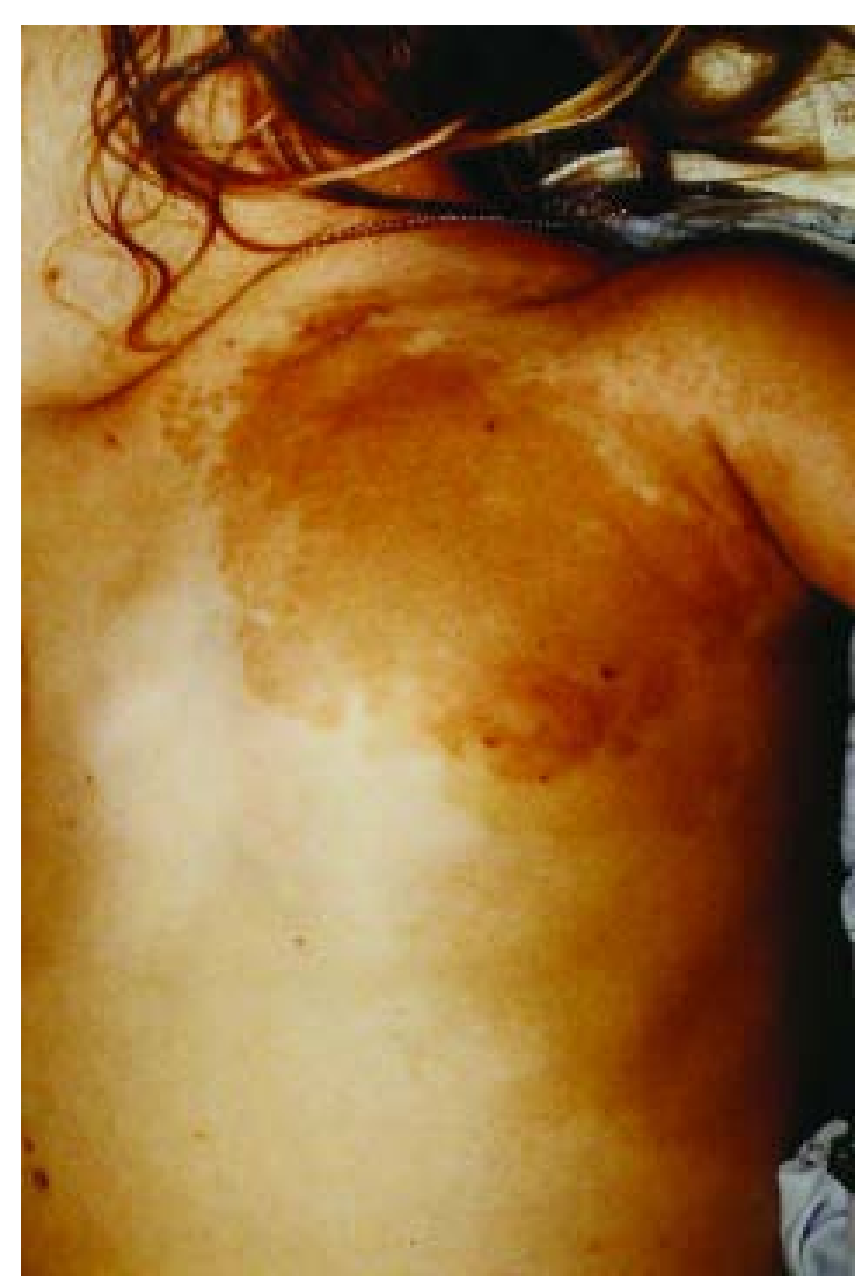

FIG. 7. Photograph of the torso of a young child illustrating skin hyperpigmentation as an element of an epineurial neurofibroma. Extension of hyperpigmentation to the midline signals neuraxis involvement.

counted for merely in terms of NF1 being a tumor suppressor gene.

Overlying Hyperpigmentation. A diffuse orange-brown coloration associated with an uneven texture of the discolored skin in the context of NF1 almost always indicates an epineurial neurofibroma with extensive skin involvement. When the hyperpigmentation approaches or reaches the midline (Fig. 7) one can be certain that the neuraxis is involved.

Pain, Tenderness, and Itching. The presence of pain in association with an epineurial neurofibroma usually means involvement of the most proximal portions of the plexuses and major nerves. Tenderness suggests nerve entrapment. Excessive itching at the site of an epineurial neurofibroma suggests an active growth period.

Overgrowth. As illustrated in Fig. 8, epineurial neurofibromas can become massive, impeding function and compromising comfort. Removing the neurofibroma and the affected limb may be a realistic choice in some circumstances. Even when they are short of massive, enlarging epineurial neurofibromas can compromise function, for

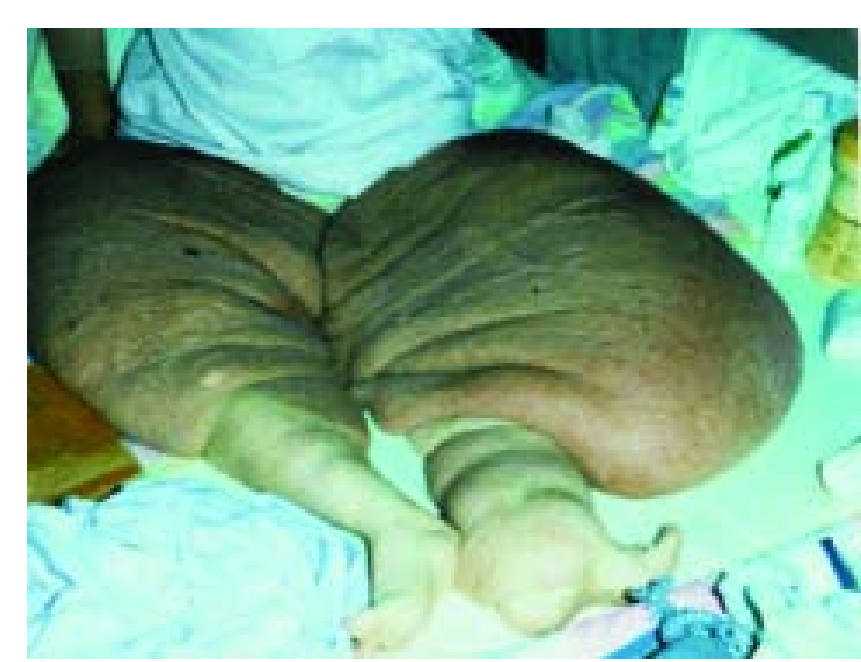

FIG. 8. Photograph of a patient with massive bilateral lower extremity epineurial neurofibromas.

example, when located on the hand, foot, or face or impinging on the birth canal.

Cosmesis. Even when modest in size, epineurial neurofibromas can compromise appearance, especially when there is overlying hyperpigmentation.

Risk for Neurofibrosarcoma. The likelihood of a neurofibrosarcoma developing in any given patient with epineurial neurofibromas is greater than $10 \%$.

\section{Neurofibrosarcomas as Part of NFI}

Neural malignancies that arise as part of the natural history of NF1 do not derive from the nerve sheath per se: they actually arise from a neurofibroma. Thus, to me, the designation "neurofibrosarcoma" is much more accurate than the appellation "malignant peripheral nerve sheath tumor" (also known by the acronym, MPNST). More accurately, per the schema portrayed in Fig. 9, the neurofibroma probably provides a microenvironment in which critical stem cells can lie dormant until they develop into a sarcoma. Again, the mature nerve sheath itself does not give rise to the malignancy. This schema is comparable to that explaining the relationship of glioblastoma multiforme to relatively benign gliomas or astrocytomas. ${ }^{18}$

In short, it is unlikely that either neurofibromas or neurofibrosarcomas derive from fully differentiated Schwann cells. Rather, the schema indicates that "immature Schwann cells" (as a specific stage in the Schwann cell lineage) give rise to neurofibromas, either on a "genetic" basis (for example, via loss of heterozygosity) or a "reactive" basis (as with miliary endoneurial neurofibromas). Neurofibrosarcomas actually arise from cells of an earlier stage, namely, "Schwann cell precursors." As has been suggested regarding glioblastomas, presumably these Schwann cell precursors are afforded a salutary microenvironment by perineurial neurofibromas and epineurial neurofibromas, eventually emerging as the cancer referred to here as neurofibrosarcoma. Parenthetically, note that, in contrast, schwannomas presumably represent a clonal proliferation of mature Schwann cells.

Neurofibrosarcomas occurring as part of NF1 are most 


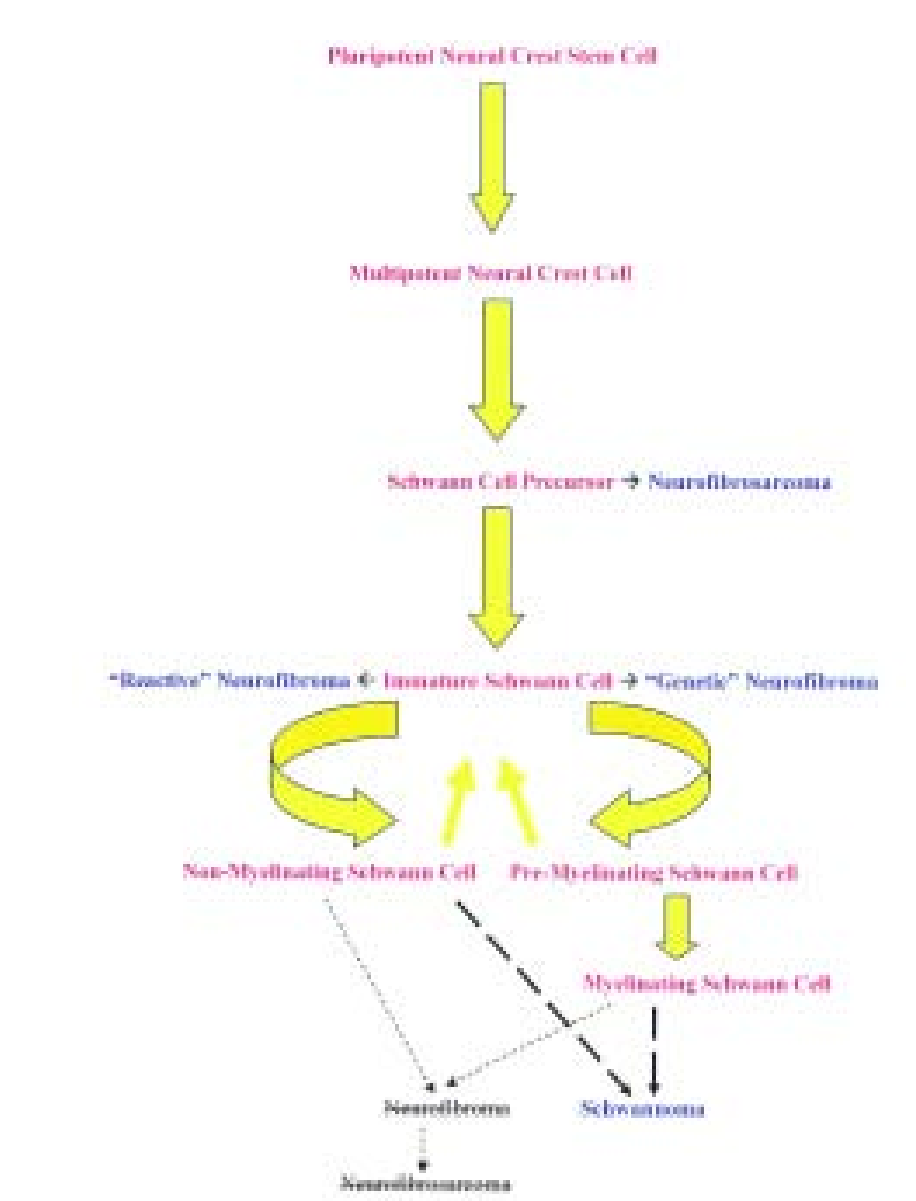

FIG. 9. Schema relating the developmental stages of the Schwann cell neural crest lineage and its relationship to the origin of neurofibromas, neurofibrosarcomas, and schwannomas.

likely to develop in the second, third, and fourth decades of life, but they may not present until the fifth or sixth decades. On the other hand, they are almost unheard of in the first decade. To date the most satisfactory treatment is amputation. If the location of the sarcoma precludes amputation, recourse to radical dissection, chemotherapy, and radiotherapy has limited benefits. Even with the most assiduous application of these modalities, death is likely to result from metastases, most often to the lung, liver, bone, and brain. As is often the case with soft tissue sarcomas, the patient often looks fine in terms of overall habitus until just weeks before loss of life. That is, there is no apparent inanition or cachexia until the very end of the disease process. This means that, even if the patient looks otherwise to be in good health, there must be a disciplined search for metastases before definitive surgery is undertaken, so that the specific goals of the surgery can be formulated accurately. Finally, there is a subgroup of patients who have a substantiated diagnosis of neurofibrosarcoma in the context of NF1 who nonetheless are apparently cured by surgical extirpation of the lesion.

The role of positron emission tomography in identifying early neurofibrosarcomas in patients with NF1 is still in the relatively early stages of development. ${ }^{2}$ However, it does look promising, although we do not have many data regarding the natural history of NF1 neurofibrosarcomas identified coincidentally by positron emission tomography or during surgery.

\section{The Neurofibroma Nerve Sheath Classification and Treatment Strategies}

\section{Surgical Treatment}

The realistic expectations concerning surgical treatment of neurofibroma must take into account the type of neurofibroma - endoneurial, perineurial, or epineurial-and the anatomical location, the extent of compromise already present at the time of surgery, and the potential adverse outcomes, including anesthesias, dysesthesias, and/or motor deficits. In general, I recommend surgery only when very specific clinical goals are anticipated, for example, minimizing pain or increasing the function of a limb and, occasionally, cosmesis. Especially for epineurial neurofibromas, amputation may become the most realistic alternative to excisions, which are almost always partial (that is, incomplete). Among the intraoperative problems associated with surgical treatment of epineurial neurofibromas is excessive blood loss, most often from small-vessel oozing. The blood loss may actually result in premature termination of the surgery. (See the discussion of ketotifen under Medical Treatment.)

From the surgical standpoint, acknowledging that only a specific type of neurofibroma will allow for clean dissection-namely the perineurial neurofibroma, whether multiplex or simplex -is a useful consequence of this article. Perineurial neurofibromas can be cleanly extirpated surgically, but with loss of function of the nerve components necessarily sacrificed. Except for amputation proximal to the lesion, it is virtually impossible to remove the entirety of an epineurial neurofibroma. The total removal of an endoneurial neurofibroma requires a very wide excision, although total removal may not be the clinical goal.

In and of itself, NF1 is not a contraindication to surgery, whether the need for surgical treatment is a result of NF1 (its features, consequences, or complications) or another condition. Nevertheless, when an adult with NF1 is to undergo surgery, the presence of a pheochromocytoma should probably be ruled out, given that this type of tumor occurs in as many as one in 200 persons with NF1.

\section{Medical Treatment}

At present, there is no standardized medical treatment of NF1 aimed at preventing or retarding the progression of neurofibromas. Oral treatment with the mast cell blocker, ketotifen, has been shown to have several beneficial effects, including the unequivocal mitigation of neurofibroma-associated pruritus and avoidance of excessive bleeding during surgical treatment of neurofibromas. ${ }^{12,13}$ Less convincing has been the influence of oral ketotifen treatment on changes in the number and/or size of the various types of neurofibromas. Unfortunately, further interest in this treatment approach languished until the demonstration by Zhang et al..$^{27}$ that mast cells are key elements of the natural history of NF1 neurofibromas, at least in knockout mice. The potential for topical treatment of endoneurial neurofibromas (and perhaps superficial epineurial neurofi- 
bromas) with mast cells blockers has been obvious for many years and is now gaining some momentum. The most dramatic effect of the oral ketotifen treatment was the marked decrease in the bleeding into the surgical wound during neurofibroma-associated surgery. ${ }^{12}$

Finally, I wish to remind the reader that one of the important consequences of identifying the category of perineurial neurofibroma is recognition of the blood-nerve barrier function of the intact perineurium. It is possible, if not likely, that at least some drugs directed at neurofibromas will not be able to cross this blood-nerve barrier and thus will be ineffective against perineurial neurofibromas.

Treatment strategies based on what is known about the NF1 gene, the neurofibromin synthesized from it, or its influence on the p21-ras pathways have not yet been fruitful. Surgery is still the mainstay of treatment.

\section{References}

1. Cardesa A, Llanes F, Furio V: Morphology of experimental tumors of the peripheral nervous system and its comparison to man. Pathol Res Pract 165:45-46, 1979

2. Ferner RE, O'Doherty MJ: Neurofibroma and schwannoma. Curr Opin Neurol 15:679-684, 2002

3. Friedman JM, Birch P, Greene C: National Neurofibromatosis Foundation International Database. Am J Med Genet 45: 88-91, 1993

4. Friedrich RE, Korf B, Funsterer C, Mautner VF: Growth type of plexiform neurofibromas in NF1 determined on magnetic resonance images. Anticancer Res 23:949-952, 2005

5. Harkin JC, Reed RJ: Tumors of the peripheral nervous system, in Atlas of Tumor Pathology. Second Series, Fascicle 3. Washington, DC: Armed Forces Institute of Pathology, 1969, pp 67-106

6. Jaakkola S, Peltonen J, Riccardi V, Chu ML, Uitto J: Type 1 neurofibromatosis: selective expression of extracellular matrix genes by Schwann cells, perineurial cells, and fibroblasts in mixed cultures. J Clin Invest 84:253-261, 1989

7. Jacks T, Shih TS, Schmitt EM, Bronson RT, Bernards A, Weinberg RA: Tumour predisposition in mice heterozygous for a targeted mutation in NF1. Nat Genet 7:353-361, 1994

8. Joseph NM, Mukouyama Y, Mosher JT, Jaegle M, Crone SA, Dormand EL, et al: Neural crest stem cells undergo multilineage differentiation in developing peripheral nerves to generate endoneurial fibroblasts in addition to Schwann cells. Development 131:5599-5612, 2004

9. Karvonen SL, Kallioinen M, Ylä-Outinen H, Peltonen J, Oikarinen A: Occult neurofibromas and increased S100 protein in the skin of patients with neurofibromatosis type 1: new insights to the etiopathomechanism of neurofibromas. Arch Dermatol 136:1207-1209, 2000

10. Kayes LM, Burke W, Riccardi VM, Bennett R, Ehrlich P, Rubenstein A, et al: Deletions spanning the neurofibromatosis 1 gene: identification and phenotype of five patients. Am J Hum Genet 54:424-436, 1994

11. Norris JF, Smith AG, Fletcher PJH, Marshall TL, Hand MJ: Neurofibromatous dermal hypoplasia: A clinical pharmacolog- ical and ultrastructural study. Br J Dermatol 112:435-441, 1985

12. Riccardi VM: A controlled multiphase trial of ketotifen to minimize neurofibroma-associated pain and itching. Arch Dermatol 129:577-581, 1993

13. Riccardi VM: Mast-cell stabilization to decrease neurofibroma growth. Preliminary experience with ketotifen. Arch Dermatol 123:1011-1016, 1987

14. Riccardi VM: Neurofibromatosis: Phenotype, Natural History and Pathogenesis, ed. 2. Baltimore: Johns Hopkins University Press, 1992

15. Riccardi VM: Trauma and wound-healing in the pathogenesis of birth defects. Proc Greenwood Genet Center 8:152-153, 1989

16. Riccardi VM, Powell PP: Denervation in von Recklinghausen's neurofibromatosis (NF-1) leads to fewer and smaller neurofibromas. Neurology 38:1810, 1988

17. Riccardi VM, Womack JE, Jacks T: Neurofibromatosis and related tumors. Natural occurrence and animal models. Am J Pathol 145:994-1000, 1994

18. Sanai N, Alvarez-Buylla A, Berger MS: Neural stem cells and the origin of gliomas. N Engl J Med 353:811-822, 2005

19. Sunderland S: The adipose tissue of peripheral nerves. Brain 68:118-122, 1945

20. Sunderland S: The connective tissue of peripheral nerves. Brain 88:841-854, 1965

21. Sunderland S, Bradley KC: Stress-strain phenomena in human peripheral nerve trunks. Brain 84:102-119, 1961

22. Sunderland S, Bradley KC: The perineurium of peripheral nerves. Anat Rec 113:125-141, 1952

23. Upadhyaya M, Huson SM, Davies M, Thomas N Chuzhanova N, Giovannini S, et al: An absence of cutaneous neurofibromas associated with a 3-bp in-frame deletion in exon 17 of the NF1 gene (c.2970-2972 delAAT): evidence of a clinically significant NF1 genotype-phenotype correlation. Am J Hum Genet 80:140-151, 2007

24. Verheijen MH, Chrast R, Burrola P, Lemke G: Local regulation of fat metabolism in peripheral nerves. Genes Dev 17: 2450-2464, 2003

25. Vinores SA, Perez-Polo JR: The effect of nerve growth factor and antibodies to nerve growth factor on ethylnitrosourea carcinogenesis in mice. J Cancer Res Clin Oncol 98:59-63, 1980

26. Viskochil D, White R, Cawthon R: The neurofibromatosis type 1 gene. Annu Rev Neurosci 16:183-205, 1993

27. Zhang Y, Taylor BR, Shannon K, Clapp DW: Quantitative effects of NF1 inactivation on in vivo hematopoiesis. J Clin Invest 108:709-715, 2001

28. Zhu Y, Ghosh P, Charnay P, Burns DK, Parada LF: Neurofibromas in NF1: Schwann cell origin and role of tumor environment. Science 296:920-922, 2002

Manuscript received March 15, 2007.

Accepted April 24, 2007.

Address reprint requests to: Vincent M. Riccardi, M.D., The Neurofibromatosis Institute, 5415 Briggs Avenue, La Crescenta, California 91214. email: riccardi@ medconsumer.com. 